\title{
Orbifold black holes
}

\author{
Muneto Nitta ${ }^{1}$, Kunihito Uzawa ${ }^{2,3, a}$ \\ ${ }^{1}$ Department of Physics, and Research and Education Center for Natural Sciences, Keio University, Hiyoshi 4-1-1, Yokohama, Kanagawa 223-8521, \\ Japan \\ 2 Department of Physics, School of Science and Technology, Kwansei Gakuin University, Sanda, Hyogo 669-1337, Japan \\ ${ }^{3}$ Research and Education Center for Natural Sciences, Keio University, Hiyoshi 4-1-1, Yokohama, Kanagawa 223-8521, Japan
}

Received: 15 February 2021 / Accepted: 26 May 2021 / Published online: 14 June 2021

(C) The Author(s) 2021

\begin{abstract}
We construct a regular black hole solution on the orbifold $\mathbb{C}^{n} / \mathbb{Z}_{n}$ in the $(2 n+1)$-dimensional EinsteinMaxwell theory. The event horizon is $S^{2 n-1} / \mathbb{Z}_{n}$.
\end{abstract}

\section{Introduction}

Black holes give profound conceptual implications for the nature of general relativity in both the classical and quantum domains. One expects that they will eventually play an equally profound role in understanding the fundamental nature of particle physics as well as general relativity. Accordingly, it is important to understand the types of black holes which can couple to matter fields. If we consider a star which undergoes gravitational collapse and form a black hole, we would expect all the matter present to be absorbed by the black hole. Since the background geometry at sufficiently late times should settle down to final state of gravitational collapse and vacuum except for presence of gauge fields associated with the black hole, it is of great interest to find all solutions of the Einstein-gauge field equations which describe stationary black holes. In four space-time dimensions, it is known that black holes are unique except for small numbers of the charges. The Kerr solution [1] has angular momentum in addition to the mass. Another simplest case is an electrically charged generalization of the Schwarzschild solution in a four-dimensional Einstein-Maxwell theory, as given by Reissner-Nordström [2-5]. When the electric charge is proportional to the mass, it is called an extremal black hole, playing important roles in string theory [6-8].

However, other than four dimensions, no such uniqueness theorem is known and thus exploring black hole solutions in higher dimensions is one of very important problems in general relativity. In fact, other than black holes, there are also black rings [9-13] for which singularity is not pointlike

\footnotetext{
a e-mail: kunihito.uzawa@gmail.com (corresponding author)
}

but ringlike. Concentrating on black holes, one of interesting class of solutions in five dimensions is given by black holes based on the Eguchi-Hanson space [14,15] found in Refs. $[16,17]$. In this case, the geometry is direct product of the time direction $\mathbb{R}$ and the Eguchi-Hanson space whose center the black hole sits on. Multiple black hole solutions were also constructed in Ref. [18]. Einstein(-Maxwell) theory with a Gauss-Bonnet term also admits similar black hole solutions $[19,20]$.

The Eguchi-Hanson space is reduced to the orbifold $\mathbb{C}^{2} / \mathbb{Z}_{2}$ in a certain limit. In other words, such an orbifold singularity is resolved for the Eguchi-Hanson space. This was generalized to the Gibbons-Hawking metric [21,22] on resolved orbifolds $\mathbb{C}^{2} / \mathbb{Z}_{n}$ or more generally asymptotically locally Euclidean (ALE) spaces. In the context of string theory, D-branes on orbifolds $\mathbb{C}^{2} / \mathbb{Z}_{n}$ were studied [23], where oribifold singularities are naturally resolved in Dbarne world-volume theories. This was generalized to higher dimensional orbifolds $\mathbb{C}^{n} / \Gamma$ with some discrete groups $\Gamma$ [24]. On the other hand, the Eguchi-Hanson space can be regarded as a Ricci-flat Kähler manifold as a cotangent bundle or complex line bundle over a sphere $S^{2}$, which is equivalent to $\mathbb{C P}^{1}$. As a higher dimensional generalization, the complex line bundle over $\mathbb{C P}^{n}$ admits a Ricci-flat Kähler metric [25-27] and this is obtained by resolving the orbifold singularity in the higher dimensional orbifold $\mathbb{C}^{n} / \mathbb{Z}_{n}$. This implies possible existence of a higher dimensional generalization of five dimensional black holes in Refs. [16,17]. In fact, the authors in Refs. [17,19,20] considered black holelike solutions based on $\mathbb{C P}^{n}$ but they are singular - a black hole singularity is not surrounded by an event horizon.

In this paper, we show that the $D(=2 n+1)$-dimensional Einstein-Maxwell theory admits regular black hole solutions on the orbifold $\mathbb{C}^{n} / \mathbb{Z}_{n}$ whose orbifold singularity is resolved, which is a complex line bundle over $\mathbb{C P}^{n}$. The black hole singularity is surrounded by an event horizon $S^{2 n-1} / \mathbb{Z}_{n}$ and thus 
it is a regular black hole. This black hole is also electrically charged, and the charge is proportional to the mass, thereby being an extremal black hole. In Sect. 2, we give an action of the Einstein-Maxwell theory. We derive solutions which describe charged black holes in this theory. The EinsteinMaxwell theory has a 2-form field strength which gives rise to charged black holes. In Sect. 3, we specialize these solutions to the cases of interest for black holes on $\mathbb{C}^{n} / \mathbb{Z}_{n}$. We will see that the case of $n=2$ reduces to previously found solutions in five dimensions $[16,17]$. The regularity at the horizon of a black hole on the orbifold is also discussed. Our solution in this paper is produced describing a black hole without naked singularity in $D(>3)$-dimensional space-time. Finally, we conclude with a discussion of our results and some implications. In appendix A we summarize some general results, including an iterative construction of real metrics on $\mathbb{C P}^{n}$, which are needed for the results in Sect. 3.

\section{D-dimensional Einstein-Maxwell theory}

In this section, we consider Einstein-Maxwell theory in $D$ dimensions. We write down the Einstein equations under a certain metric ansätze, which is a generalization of those of known static 5-dimensional solutions. After we then solve the Einstein equations and present the solutions explicitly, we compare the results of $D$-dimensional solutions with 5 dimensional one.

Let us consider a gravitational theory with the metric $g_{M N}$, and 2-form field strength $F_{(2)}=d A_{(1)}$, coming from 1-form gauge potential $A_{(1)}$. The action for the Einstein-Maxwell theory is written as

$S=\frac{1}{2 \kappa^{2}} \int d^{D} x \sqrt{-g}\left[R-\frac{1}{2 \cdot 2 !} F_{(2)}^{2}\right]$,

where $\kappa^{2}$ is the $D$-dimensional gravitational constant.

The field equations are given by

$$
\begin{aligned}
& R_{M N}=\frac{1}{2 \cdot 2 !}\left[2 F_{M A} F_{N}{ }^{A}-\frac{1}{D-2} g_{M N} F_{(2)}^{2}\right], \\
& d\left[* F_{(2)}\right]=0
\end{aligned}
$$

where $*$ is the Hodge dual operator in the $D$-dimensional spacetime.

We look for solutions whose spacetime metric has the form

$d s^{2}=-h^{-2}(y) d t^{2}+h^{\frac{2}{D-3}}(y) u_{i j}(\mathrm{Y}) d y^{i} d y^{j}$,

where $u_{i j}(\mathrm{Y})$ is the metric of the $(D-1)$-dimensional space Y which depends only on the $(D-1)$-dimensional coordinates $y^{i}$, and the function $h$ depends only on $y^{i}$. We also assume that the gauge field strength $F_{(2)}$ is given by
$F_{(2)}= \pm \sqrt{2}\left(1+\frac{1}{D-3}\right)^{1 / 2} d\left(h^{-1}\right) \wedge d t$.

Under the assumptions given above, we first reduce the gauge field equations other than the Einstein equations to a simple set of equations. For the 2-form field strength $F_{(2)}=d A_{(1)}$, it implies that $F_{(2)}$ is a closed form and the Bianchi identity is automatically satisfied. Also the equation of motion for the gauge field becomes

$\triangle_{\mathrm{Y}} h=0$,

where $\Delta_{\mathrm{Y}}$ is the Laplacian with respect to the metric $u_{i j}(\mathrm{Y})$. In order to complete the system of equations, we also have to consider the Einstein equations (2a). Under our ansatz, these equations become

$$
\begin{aligned}
& h^{-3-\frac{2}{D-3}} \triangle_{\mathrm{Y}} h=0, \\
& R_{i j}(\mathrm{Y})-\frac{1}{D-3} h^{-1} u_{i j} \triangle_{\mathrm{Y}} h=0 .
\end{aligned}
$$

In terms of Eq. (2b), Eq. (6) are equivalent to [28]

$$
R_{i j}(\mathrm{Y})=0, \quad \triangle_{\mathrm{Y}} h=0 .
$$

\section{Black holes on the orbifold $\mathbb{C}^{n} / \mathbb{Z}_{n}$}

Now we apply the general formulation developed in the last section to the space which is described as a complex line bundle over $\mathbb{C P}^{n-1}$ in order to find a generalization of the solution discussed in Ref. [16]. The metric $u_{i j}(\mathrm{Y})$ on the (2n)-dimensional space $\mathrm{Y}$ in Eq. (3) is thus given by

$$
\begin{aligned}
& u_{i j}(\mathrm{Y}) d y^{i} d y^{j}=d r^{2} \\
& +r^{2}\left[\left\{d \rho+\sin ^{2} \xi_{n-1}\left(d \psi_{n-1}+\frac{1}{2(n-1)} \omega_{n}\right)\right\}^{2}+d s_{\mathbb{C P}^{n-1}}^{2}\right],
\end{aligned}
$$

where $r$ is a radial coordinate, $\rho$ is a coordinate of $S^{1}, \xi_{n-1}$ and $\psi_{n-1}$ are coordinates of the $\mathbb{C P}^{n-1}$ space with the ranges $0 \leq \xi_{n-1} \leq \pi / 2,0 \leq \psi_{n-1} \leq 2 \pi$, and $\omega_{n-1}$ and $d s_{\mathbb{C P}^{n-1}}^{2}$ denote a one-form and a metric on the $\mathbb{C P}^{n-1}$ space, respectively, recursively defined as $[17,19,20]$

$$
\begin{aligned}
& d s_{\mathbb{C P}^{n-1}}^{2} \\
& =2 n\left[d \xi_{n-1}^{2}+\sin ^{2} \xi_{n-1} \cos ^{2} \xi_{n-1}\left\{d \psi_{n-1}+\frac{1}{2(n-1)} \omega_{n-2}\right\}^{2}\right. \\
& \left.\quad+\frac{1}{2(n-1)} \sin ^{2} \xi_{n} d s_{\mathbb{C P} n-2}^{2}\right],
\end{aligned}
$$

and

$$
\omega_{n-2}=2(n-1) \sin ^{2} \xi_{n-2}\left[d \psi_{n-2}+\frac{1}{2(n-2)} \omega_{n-3}\right],
$$$$
d s_{\mathbb{C P}^{1}}^{2}=4\left(d \xi_{1}^{2}+\sin ^{2} \xi_{1} \cos ^{2} \xi_{1} d \psi_{1}^{2}\right),
$$

$$
\omega_{1}=4 \sin ^{2} \xi_{1} d \psi_{1} \text {. }
$$


Here, $(r, \rho)$ denotes a complex line, and $\rho$ together with $\mathbb{C P}^{n}$ describe a $2 n-1$ dimensional sphere $S^{2 n-1} / \mathbb{Z}_{n}=S^{D-2} / \mathbb{Z}_{n}$, which is actually an event horizon.

Let $h$ be a function on $\mathrm{Y}$ of the form:

$h=h(r)$.

The remaining non-trivial equation (7) reduces to

$\Delta_{\mathrm{Y}} h=\frac{1}{r^{D-2}}\left(r^{D-2} h^{\prime}\right)^{\prime}=0$.

Thus, the solution for $h$ under our ansatz is given by

$h(r)=c_{0}+\frac{c_{1}}{r^{D-3}}$,

where $c_{0}$ and $c_{1}$ are constants. The constant of integration $c_{0}$ has been set equal to 1 in the following, which is the condition required for having an asymptotically flat geometry at $r \rightarrow \infty$, while the integration constant $c_{1}$ in Eq. (13) sets the mass scale of the solution; it has been taken to be positive in order to ensure the absence of naked singularities at finite $r$.

The metric of a spherically symmetric, charged black hole is thus:

$$
\begin{aligned}
d s^{2}= & -\left(1+\frac{c_{1}}{r^{D-3}}\right)^{-2} d t^{2} \\
& +\left(1+\frac{c_{1}}{r^{D-3}}\right)^{\frac{2}{D-3}} u_{i j}(\mathrm{Y}) d y^{i} d y^{j} .
\end{aligned}
$$

While we compute the physical charge in terms of the solution (14)

$$
\begin{aligned}
Q & =\frac{1}{2 \kappa^{2}} \int_{S^{D-2} / \mathbb{Z}_{n}} * F_{(2)} \\
& = \pm \sqrt{2(D-2)(D-3)} \frac{c_{1} V_{S^{D-2} / \mathbb{Z}_{n}}}{2 \kappa^{2}},
\end{aligned}
$$

we can also derive the expression of ADM mass and compare it with the physical charge

$M=(D-2) \frac{c_{1} V_{S^{D-2} / \mathbb{Z}_{n}}}{\kappa^{2}}=\sqrt{\frac{2(D-2)}{D-3}}|Q|$,

where $V_{S^{D-2}} / \mathbb{Z}_{n}$ denotes the volume of $S^{D-2} / \mathbb{Z}_{n}$. Since the mass is proportional to the charge, this is an extremal black hole.

One notes that there are some interesting remarks about the solution we have found. First, one arises when we note that the function $h$ which characterizes the solution diverges at $r=0$. For the region of spacetime determined by $r=0$ to coincide with the origin of the spherical coordinates, and thus with the location of the charged object, a necessary condition is that the radius of the $(D-2)$-sphere $S^{D-2}$ shrinks to zero size there. Now the radius of the $S^{D-2}$ in the geometry (14) is given by $h^{1 /(D-3)} r$. If we consider qualitatively that the function $h$ behaves like $h \sim r^{-(D-3)}$ near $r=0$, that $h^{1 /(D-3)} r \sim$ constant.
One would like to know whether the locus $r=0$ is a singularity of the geometry or not. When this actually can be checked using the components of the Riemann tensor and the square of field strength,

$$
\begin{aligned}
& \lim _{r \rightarrow 0} R_{M N P Q} R^{M N P Q} \\
& =-2 c_{1}^{2-\frac{4}{D-3}}\left[4 D^{4}-41 D^{3}+154 D^{2}-251 D+149\right], \\
& \lim _{r \rightarrow 0} F_{M N} F^{M N}=-\frac{2(D-2)}{D-3} c_{1}^{\frac{2}{D-3}},
\end{aligned}
$$

the invariant does not diverge at $r=0$. This in turn implies the non-divergence of the square of the Riemann tensor. We may thus conclude that for the charged black hole, the curvature does not diverge at the location of the charged object itself. This could have been guessed from the fact that, for instance, the energy of gauge field is not singular at $r=0$, and provides a regular source term to the Einstein equations. However, in order to examine the regularity at the horizon $r=0$, this conclusion overlooks the study of the Riemann curvature measured by a free-falling observer with orthonormal bases [17]. Before drawing conclusions on the physical consequences of this regular behaviour, we should consider the regularity of the black hole solution in more detail. We will discuss the regularity of the null hypersurface and show the metric components and the Riemann curvature components near $r=0$ where is the horizon. Then we discuss an analytic extension across the $r=0$ surface in our solution. Since the metric (14) is apparently singular null surface at $r=0$, we will present the coordinates so that the surface becomes smooth.

To see that the $r=0$ surface is smooth, let us here introduce the new coordinates $(v, \tilde{r})$

$d v=d t+h^{(D-2) /(D-3)} d r, \quad d \tilde{r}=r^{(D-4)} d r$.

The line element turns out to be

$$
\begin{aligned}
d s^{2}= & -h^{-2} d v^{2}+h^{-\frac{D-4}{D-3}} \tilde{r}^{-(D-4)} d v d \tilde{r} \\
& +h^{\frac{2}{D-3}} u_{i j}(\mathrm{Y}) d y^{i} d y^{j}
\end{aligned}
$$

These coordinates clearly describe smooth functions of $r$, namely giving an analytic extension of the metric (19):

$$
\begin{aligned}
g_{v \tilde{r}} & =c_{1}^{-\frac{D-4}{D-3}}\left[1-\frac{D-4}{c_{1}(D-3)} r^{D-3}\right]+O\left(r^{2(D-3)}\right) \\
& =c_{1}^{-\frac{D-4}{D-3}}\left(1-\frac{D-4}{c_{1}} \tilde{r}\right)+O\left(\tilde{r}^{2}\right), \\
g_{i j} & =c_{1}^{\frac{2}{D-3}}\left[1+\frac{2}{c_{1}(D-3)} r^{D-3}\right]+O\left(r^{2(D-3)}\right) \\
& =c_{1}^{\frac{2}{D-3}}\left(1+\frac{2}{c_{1}} \tilde{r}\right)+O\left(\tilde{r}^{2}\right) .
\end{aligned}
$$


Since these components do not have any fractional power of $\tilde{r}$, it implies the null hypersurface at $r=0$ becomes regular.

The aim of the rest of this section is to discuss the behavior of the geometry near $r=0$ in terms of the computation of the expansion for an outgoing null geodesic congruence. Now we compute the curvature for a particular metric to present an analytic extension for the background (19) [17]. The vielbeins are determined with the metric (19):

$e_{0}^{(0)}=-1, \quad e_{1}^{(0)}=-h^{\frac{1}{2 n}} \sqrt{h^{2}-1}$,

$e_{0}^{(1)}=-h^{-1} \sqrt{h^{2}-1}$,

$e_{1}^{(1)}=-h^{1+\frac{1}{2 n}}, \quad e_{i}^{(\alpha)}=h^{\frac{1}{2 n}} r \bar{e}_{i}^{(\alpha)}$.

Here we choose $e^{(0) \mu} \partial_{\mu}$ as a geodesic tangent vector in the radial direction, $\alpha$ runs over the $(D-2)$ space values $1, \ldots$, $(D-2)$, and the vielbeins obey

$$
\begin{aligned}
& e^{(0) \mu} \nabla_{\mu} e^{(A) v}=0, \quad g^{\mu \nu} e_{\mu}^{(A)} e_{\nu}^{(B)}=\eta^{(A)(B)}, \quad u^{i j} \bar{e}_{i}^{(\alpha)} \bar{e}_{j}^{(\beta)} \\
& \quad=\delta^{(\alpha)(\beta)},
\end{aligned}
$$

where $\nabla_{\mu}$ denotes the covariant derivative with respect to the $D$-dimensional metric $g_{\mu \nu}, \delta^{(\alpha)(\beta)}$ implies $(D-2) \times(D-2)$ unit matrix and

$\eta^{(A)(B)}=\eta_{(A)(B)}=\operatorname{diag}(-1,1, \ldots, 1)$.

The expansion for an outgoing null geodesic congruence $k^{\mu}$ is thus following [17]

$\theta_{+}=h^{\mu \nu} \nabla_{\mu} k_{v}$

where $h^{\mu \nu}$ is defined by

$h^{\mu v}=g^{\mu v}+k^{\mu} \ell^{v}+\ell^{\mu} k^{v}$,

and the null vector fields $k^{\mu}, \ell^{\mu}$ satisfy

$k^{\mu} k_{\mu}=\ell^{\mu} \ell_{\mu}=0, \quad k^{\mu} \ell_{\mu}=-1$.

In terms of vielbeins (21), we take outgoing and ingoing null vector fields $k^{\mu} \partial_{\mu}$ and $\ell^{\mu} \partial_{\mu}$ by

$$
\begin{aligned}
& k^{\mu} \partial_{\mu}=\frac{1}{\sqrt{2}}\left[e^{(0) \mu}-e^{(1) \mu}\right] \partial_{\mu}=\frac{h^{2}\left(1-h^{-1} \sqrt{h^{2}-1}\right)}{\sqrt{2}} \partial_{t} \\
& -\frac{h^{-\frac{1}{D-3}}\left(\sqrt{h^{2}-1}-h\right)}{\sqrt{2}} \partial_{r}, \\
& \ell^{\mu} \partial_{\mu}=\frac{1}{\sqrt{2}}\left[e^{(0) \mu}+e^{(1) \mu}\right] \partial_{\mu}=\frac{h^{2}\left(1+h^{-1} \sqrt{h^{2}-1}\right)}{\sqrt{2}} \partial_{t} \\
& -\frac{h^{-\frac{1}{D-3}}\left(\sqrt{h^{2}-1}+h\right)}{\sqrt{2}} \partial_{r},
\end{aligned}
$$

Since the field $h^{\mu v}$ vanishes at $r \geq 0$ with vielbeins given in Eq. (21), the expansion also becomes zero for $r \geq 0$.
Hence, the hypersurface at $r=0$ looks like the apparent horizon for observers in the region $r \geq 0$.

We now proceed to the discussion of an analytic extension across the horizon $r=0$ briefly, and compute the Riemann curvature measured by a free-falling observer with orthonormal bases. the orthonormal frame in the limit $r \rightarrow 0$ are:

$$
\begin{aligned}
R^{(0)(1)(0)(1)} & =c_{1}^{-\frac{2}{D-3}}(D-3)^{2}+O\left(r^{2(D-3)}\right) \\
R^{(0)(\alpha)(0)(\beta)} & =O\left(r^{2(D-3)}\right) \\
R^{(1)(\alpha)(1)(\beta)} & =O\left(r^{2(D-3)}\right) \\
R^{(\alpha)(\beta)(\gamma)(\delta)} & =c_{1}^{\frac{4}{D-3}}\left(1+\frac{4}{D-3} \frac{r^{D-3}}{c_{1}}\right) \delta^{\alpha \beta}+O\left(r^{2(D-3)}\right) .
\end{aligned}
$$

The other components of the Riemann curvature vanish. These components show that the solution is not singular at $r=0$ for $D>3$. Hence we conclude that the horizon $r=0$ is smooth and there is $\mathrm{C}^{2}$ extension across the $r=0$ surface. This is similar with the result that the singularity at the horizon disappears for the Reissner-Nordström metrics.

\section{Discussions}

We conclude with some comments on the properties of the solutions we have discussed in this letter, and potential applications. We have discussed exact solutions in $D$-dimensional Einstein-Maxwell theory since this is the generalization of interest for black hole solutions. The space-time structure of the geometry has been analyzed, and one finds that the locus $r=0$ is a regular horizon.

The solutions given here display explicitly the property that the expansion of an outgoing null geodesic congruence vanishes. It is therefore likely that there are null hypersurfaces. The examination of our solution for black holes on an orbifold suggested that these are nonsingular solutions because the spacetimes look like black holes for an observer outside the hypersurface. The essential point is that not only the Kretschmann invariant but the Riemann curvature in a frame of an observer parallelly transported along a free-fall geodesic becomes finite for $D>3$. Hence, the solution does not have any curvature singularity at the null hypersurface. This result leads one to conclude that the observer along the free-fall geodesic can across the horizon due to the $\mathrm{C}^{2}$ extension traversing there.

The existence of the electric charge is essential in our solutions because they are extremal black holes. It is an open question whether there exist (non-extremal) black hole solutions without an electric charge. It is also interesting problem if our solutions can be embedded into supergravity or superstring. Since our solutions are extremal, it is likely the case that they are BPS states preserving a fraction of supersymmetry. 
The case of $n=2$ reduces to $D=5$ dimensional black holes on the resolved orbifold $\mathbb{C}^{2} / \mathbb{Z}_{2}[16,17]$. In this case, multiple black hole solutions were also constructed in Ref. [18], which should correspond to the orbifold $\mathbb{C}^{2} / \mathbb{Z}_{k+1}$ for $k$ black holes. Constructing multi-black holes in general dimensions remains as one of future problems.

As a further generalization, one could replace the $\mathbb{C P}^{n-1}$ manifold by other homogeneous Kähler manifolds since Ricci-flat metrics on homogenous Kähler manifolds are known [25-27] including conifolds [29,30]. One would then obtain higher dimensional black holes with event horizons of various topologies.

One can clearly modify the derivation in this paper to obtain higher-dimensional charged objects in any dimension larger than four.

It is thus an interesting question whether or not there exist corresponding black $p$-branes in $D$ dimensions [31,32]. Although we have not dwelt upon the aspect of this subject in this paper, the reader is referred to Ref. [33] for a treatment of some generalization to black $p$-brane solutions. These includes also $p$-branes on orbifolds, and can involve fractional $p$-branes which are stucked at orbifold singularities [23,24,34-37].

There is an issue to consider the black holes in expanding universe. It was pointed out in Refs. [38,39] that we can generalize the extremal solutions to time-dependent background, while it was noted that the non-extremal solutions cannot be extended to a time-dependent solution [39].

Acknowledgements We thank H. Ishihara, M. Kimura, and S. Tomizawa for useful comments. The work of M.N. is supported in part by Grant-in-Aid for Scientific Research, JSPS KAKENHI Grant Number JP18H01217. The work of K. U. is supported by Grants-in-Aid from the Scientific Research Fund of the Japan Society for the Promotion of Science, under Contract no. 16K05364. This work was supported by the Ministry of Education, Culture, Sports, Science (MEXT-)Supported Program for the Strategic Research Foundation at Private Universities "Topological Science" (Grant no. S1511006).

Data Availability Statement This manuscript has associated data in a data repository. [Authors' comment: The datasets generated during and/or analysed during the current study are available from the corresponding author on reasonable request.]

Open Access This article is licensed under a Creative Commons Attribution 4.0 International License, which permits use, sharing, adaptation, distribution and reproduction in any medium or format, as long as you give appropriate credit to the original author(s) and the source, provide a link to the Creative Commons licence, and indicate if changes were made. The images or other third party material in this article are included in the article's Creative Commons licence, unless indicated otherwise in a credit line to the material. If material is not included in the article's Creative Commons licence and your intended use is not permitted by statutory regulation or exceeds the permitted use, you will need to obtain permission directly from the copyright holder. To view a copy of this licence, visit http://creativecomm ons.org/licenses/by/4.0/.

Funded by SCOAP ${ }^{3}$.

\section{Appendix}

\section{Appendix A: Product of $\mathbb{C P}^{n-1}$ space and their U(1) bun- dles}

In this section, we discuss the Ricci flat space on $\mathbb{C P}^{n-1}$. Let us summarize the Fubini-Study construction of the EinsteinKahler metric on $\mathbb{C P}^{n-1}$. We introduce the complex coordinates $z^{M}$ on $\mathbb{C}^{n}$, with the flat metric

$d s_{\mathbb{C}^{n}}^{2}=d z^{M} d \bar{z}_{M}$,

and inhomogeneous coordinates $\zeta^{m}=z^{m} / z^{0}$, in the patch where $z^{0} \neq 0$. Here we split the index $M$ into $M=(0, \mu)$, and $1 \leq \mu \leq n-1$.

If we use [40]

$z^{0}=\mathrm{e}^{i \tau}\left|z^{0}\right|, \quad r=\sqrt{z^{\mu} \bar{z}_{\mu}}, \quad f=1+\zeta^{\mu} \bar{\zeta}^{\bar{\mu}}$,

the flat metric on $\mathbb{C}^{n}$ can be written by

$d s_{\mathbb{C}^{n}}^{2}=d r^{2}+r^{2} d \Omega_{2 n-1}$,

where $d \Omega_{2 n-1}$ denotes the metric of the $S^{2 n-1}$ [40]

$$
\begin{aligned}
d \Omega_{2 n-1} & =(d \tau+A)^{2}+f^{-1} d \zeta^{\mu} d \bar{\zeta}^{\bar{\mu}}-f^{-2} \bar{\zeta}^{\bar{\mu}} \zeta^{\nu} d \zeta^{\mu} d \bar{\zeta}^{\bar{\nu}}, \\
A & =\frac{i}{2} f^{-1}\left(\zeta^{\mu} d \bar{\zeta}^{\bar{\mu}}-\bar{\zeta}^{\bar{\mu}} d \zeta^{\mu}\right) .
\end{aligned}
$$

Since the metric (A4) denotes the unit $S^{2 n-1}$ which is described as a $\mathrm{U}(1)$ bundle over $\mathbb{C} \mathrm{P}^{n-1}$, it contains the FubiniStudy metric $d s_{\mathrm{FS}}^{2}$ on $\mathbb{C P}^{n-1}$

$d s_{\mathrm{FS}}^{2}=f^{-1} d \zeta^{\mu} d \bar{\zeta}^{\bar{\mu}}-f^{-2} \bar{\zeta}^{\bar{\mu}} \zeta^{v} d \zeta^{\mu} d \bar{\zeta}^{\bar{\nu}}$,

and is also referred as the unit $\mathbb{C P}{ }^{n-1}$ metric. The metric (A4) thus corresponds to the hopf fibration of the unit $(2 n-1)$ sphere. For example, if we take $n=3$, we get the metric of $\mathbb{C P}^{2}$ space $[22,41]$ :

$$
\begin{aligned}
d s_{\mathbb{C P}}^{2}= & \left(1+\rho^{2}\right)^{-2} d \rho^{2}+\frac{\rho^{2}}{4}\left(1+\rho^{2}\right)^{-2}(d \psi+\cos \theta d \phi)^{2} \\
& +\frac{\rho^{2}}{4}\left(1+\rho^{2}\right)^{-1}\left(d \theta^{2}+\sin ^{2} \theta d \phi^{2}\right),
\end{aligned}
$$

where we introduce coordinates by defining Euler angles $(\psi, \theta, \phi)$ and a radial coordinate $\rho$ [41]

$\zeta^{1}=\rho \cos \left(\frac{\theta}{2}\right) \mathrm{e}^{i(\psi+\phi) / 2}$,
$\zeta^{2}=\rho \sin \left(\frac{\theta}{2}\right) \mathrm{e}^{i(\psi-\phi) / 2}$.

There are coordinate singularities at $\rho=0$ and $\theta=0$ or $\pi$ if we set

$0 \leq \theta \leq \pi, \quad 0 \leq \phi \leq 2 \pi, \quad 0 \leq \psi \leq 4 \pi, \quad 0 \leq \rho \leq \infty$. 


\section{References}

1. R.P. Kerr, Gravitational field of a spinning mass as an example of algebraically special metrics. Phys. Rev. Lett. 11, 237 (1963)

2. H. Reissner, Uber die Eigengravitation des elektrischen Feldes nach der Einsteinschen Theorie. Annalen der Physik (in German) 50, 106-120 (1916)

3. H. Weyl, Zur Gravitationstheorie. Annalen der Physik (in German) 54, 117-145 (1917)

4. G. Nordström, On the energy of the gravitational field in Einstein's theory. Verhandl. Koninkl. Ned. Akad. Wetenschap. Afdel. Natuurk. 26, 1201-1208 (1918)

5. G.B. Jeffery, The field of an electron on Einstein's theory of gravitation. Proc. R. Soc. Lond. A 99, 123-134 (1921)

6. P.K. Townsend, Black holes: lecture notes, arXiv:gr-qc/9707012

7. K.S. Stelle, in Trieste 1997, High energy physics and cosmology, p. 29-127 arXiv:hep-th/9803116

8. M.J. Duff, arXiv:hep-th/9912164

9. R. Emparan, H.S. Reall, A rotating black ring solution in five-dimensions. Phys. Rev. Lett. 88, 101101 (2002). arXiv:hep-th/0110260

10. H. Elvang, A Charged rotating black ring. Phys. Rev. D 68, 124016 (2003). arXiv:hep-th/0305247

11. H. Elvang, R. Emparan, D. Mateos, H.S. Reall, A supersymmetric black ring. Phys. Rev. Lett. 93, 211302 (2004). arXiv:hep-th/0407065

12. R. Emparan, H.S. Reall, Black rings. Class. Quantum Gravity 23, R169 (2006). arXiv:hep-th/0608012

13. I. Bena, N.P. Warner, Black holes, black rings and their microstates. Lect. Notes Phys. 755, 1 (2008). arXiv:hep-th/0701216

14. T. Eguchi, A.J. Hanson, Asymptotically flat Selfdual solutions to Euclidean gravity. Phys. Lett. B 74, 249-251 (1978)

15. T. Eguchi, P.B. Gilkey, A.J. Hanson, Gravitation, gauge theories and differential geometry. Phys. Rep. 66, 213 (1980)

16. H. Ishihara, M. Kimura, K. Matsuno, S. Tomizawa, Black holes on Eguchi-Hanson space in five-dimensional Einstein-Maxwell theory. Phys. Rev. D 74, 047501 (2006). arXiv:hep-th/0607035

17. T. Tatsuoka, H. Ishihara, M. Kimura, K. Matsuno, Extremal charged black holes with a twisted extra dimension. Phys. Rev. D 85, 044006 (2012). arXiv:1110.6731 [hep-th]

18. H. Ishihara, M. Kimura, K. Matsuno, S. Tomizawa, Kaluza-Klein multi-black holes in five-dimensional Einstein-Maxwell theory. Class. Quantum Gravity 23, 6919 (2006). arXiv:hep-th/0605030

19. M.H. Dehghani, R.B. Mann, NUT-charged black holes in Gauss-Bonnet gravity. Phys. Rev. D 72, 124006 (2005). arXiv:hep-th/0510083

20. M.H. Dehghani, S.H. Hendi, Taub-NUT/bolt black holes in Gauss-Bonnet-Maxwell gravity. Phys. Rev. D 73, 084021 (2006). arXiv:hep-th/0602069

21. G.W. Gibbons, S.W. Hawking, Gravitational multi-instantons. Phys. Lett. B 78, 430 (1978)

22. G.W. Gibbons, S.W. Hawking, Classification of gravitational instanton symmetries. Commun. Math. Phys. 66, 291-310 (1979)
23. M.R. Douglas, G.W. Moore, D-branes, quivers, and ALE instantons, arXiv:hep-th/9603167

24. M.R. Douglas, B.R. Greene, D.R. Morrison, Orbifold resolution by D-branes. Nucl. Phys. B 506, 84-106 (1997). arXiv:hep-th/9704151

25. K. Higashijima, T. Kimura, M. Nitta, Ricci flat Kahler manifolds from supersymmetric gauge theories. Nucl. Phys. B 623, 133 (2002). arXiv:hep-th/0108084

26. K. Higashijima, T. Kimura, M. Nitta, Gauge theoretical construction of noncompact Calabi-Yau manifolds. Ann. Phys. 296, 347370 (2002). arXiv:hep-th/0110216

27. K. Higashijima, T. Kimura, M. Nitta, Calabi-Yau manifolds of cohomogeneity one as complex line bundles. Nucl. Phys. B 645, 438 (2002). arXiv:hep-th/0202064

28. R.C. Myers, Higher dimensional black holes in compactified spacetimes. Phys. Rev. D 35, 455 (1987)

29. K. Higashijima, T. Kimura, M. Nitta, Supersymmetric nonlinear sigma models on Ricci flat Kahler manifolds with $\mathrm{O}(\mathrm{N})$ symmetry. Phys. Lett. B 515, 421-425 (2001). arXiv:hep-th/0104184

30. K. Higashijima, T. Kimura, M. Nitta, A note on conifolds. Phys. Lett. B 518, 301 (2001). arXiv:hep-th/0107100

31. H. Lu, C.N. Pope, E. Sezgin, K.S. Stelle, Stainless super $p$-branes. Nucl. Phys. B 456, 669 (1995). arXiv:hep-th/9508042

32. R. Argurio, F. Englert, L. Houart, Intersection rules for $p$-branes. Phys. Lett. B 398, 61 (1997). arXiv:hep-th/9701042

33. M. Nitta, K. Uzawa, Fractional black $p$-branes on orbifold $\mathbb{C}^{n} / \mathbb{Z}_{n}$. JHEP 2103, 018 (2021). arXiv:2012.13285 [hep-th]

34. H. Nakajima, Moduli spaces of anti-self-dual connections on ALE gravitational instantons. Invent. Math. 102, 267 (1990)

35. P.B. Kronheimer, H. Nakajima, Yang-Mills instantons on ALE gravitational instantons. Math. Ann. 288, 263 (1990)

36. T. Kimura, M. Nitta, Vortices on orbifolds. JHEP 09, 118 (2011) arXiv:1108.3563 [hep-th]

37. M. Eto, Y. Isozumi, M. Nitta, K. Ohashi, K. Ohta, N. Sakai, Dbrane construction for non-Abelian walls. Phys. Rev. D 71, 125006 (2005). arXiv:hep-th/0412024

38. P. Binetruy, M. Sasaki, K. Uzawa, Dynamical D4-D8 and D3D7 branes in supergravity. Phys. Rev. D 80, 026001 (2009). arXiv:0712.3615 [hep-th]

39. K.I. Maeda, N. Ohta, K. Uzawa, Dynamics of intersecting brane systems: classification and their applications. JHEP 0906, 051 (2009). arXiv:0903.5483 [hep-th]

40. P. Hoxha, R.R. Martinez-Acosta, C.N. Pope, Kaluza-Klein consistency, killing vectors, and Kahler spaces. Class. Quantum Gravity 17, 4207 (2000). arXiv:hep-th/0005172

41. G.W. Gibbons, C.N. Pope, $\mathrm{CP}^{2}$ as a gravitational instanton. Commun. Math. Phys. 61, 239 (1978) 